formally established if the animals would have been protected from rechallenge with SIV grown in human cells, but in previous experiments we have obtained protection following reboosting.

At first sight our results suggest that the challenge viruses used in these experiments may have acquired properties, either by genetic selection or, perhaps more likely, epigenetically, from the cells in which they were grown. Thus anti-cellular responses elicited by immunization may have been responsible for the protection observed when both vaccine and challenge virus were derived from the xenogeneic human cells. But caution is necessary in interpreting these results: because the virus used for rechallenge was produced in rhesus monkey PBMC from the original SIV $_{\text {MAC }} 251$ stock, it could have contained variants that were critically divergent from the cognate virus used to prepare the vaccine.

It is indeed remarkable that crude inactivated SIV vaccines provide complete protection against challenge with various antigenically distinct viruses which, in unvaccinated animals, invariably induce AIDS. However, this failure to protect against virus grown in monkey PBMC and the significance of anticellular responses must be understood so that the protecting machanisms can be defined and applied to AIDS vaccine development.

M. P. CRanage

L. A. E. ASHWORTH

P. J. GREENAWAY

PHLS Centre for Applied Microbiology and Research,

Porton Down.

Salisbury SP4 OJG, UK

M. MURPHEY-CORB

Delta Regional Primate Research

Center

Tulane University,

Covington,

Louisianna 70434, USA

R. C. DESROSIERS

New England Regional Primate

Research Center,

One Pine Drive,

Southborough,

Massachusetts 01772, USA

\section{Temperature time-series?}

SIR - Ghil and Vautard ${ }^{1}$ applied the method of singular-spectrum analysis to a 135-year time-series of global annualaverage near-surface temperatures, and found a pair of high-variance empirical orthogonal functions (EOFs) characterizing a temperature oscillation with a period of slightly more than 20 years. Elsner and Tsonis ${ }^{2}$ repeated the analysis considering the most recent 130-, 110- a, Portion of spectrum of covariance matrix constructed $^{1,2}$ from $M=30$ lagged copies of the IPCC consensus time-series of global temperatures ${ }^{3}$. Note the linear scale, to emphasize the similarity of eigenvalues 4,5 and 6 in all cases considered. Ordinates: $a$, eigenvalue; $b, c$, eigenvector. $b$, EOFs 3-6 for case 2: $N=110$ years, no averaging of covariance matrix elements. Note EOFs 3 (dotted line) and 6 (dashed line) are in quadrature, indicating a 20-30-year oscillation, while EOFs 4 (solid line) and 5 (dashdot line) indicate a 5-6 year oscillation. $c$, EOFs $3-6$ for case 2: $N=110$ years, averaging covariance matrix elements along diagonals. Note EOFs 3 and 4 are now in quadrature.

and 90 -year segments of the time-series. They report that the EOF 'signature' of the 20-year oscillation appears only if all 130 years are included, suggesting it may be an artefact of the (unreliable) early part of the time-series.

We found this pair of results intriguing, because Ghil and Vautard also show a reconstruction of the original data by projection onto a number of subsets of their EOF eigenbasis, including one in which only the lowest 4 EOFs (which include the 20-year oscillatory pair) are retained (their Fig. 3a). The amplitude of the 20-year oscillation does not appear to diminish in the later part of the time-series, as would be expected if the conclusion of ref. 2 (that all the power in this pair of EOFs is confined to the earlier part) were correct. Using the IPCC consensus time-series ${ }^{3}$ (data kindly provided by D. E. Parker), we reproduced both sets of results. Are they a paradox?

The answer is no. If we consider the relevant portion of the spectrum of the lag-covariance matrix, including all 130 years ( $a$ in the figure, case 1), we notice that the eigenvalues of EOFs 4,5 and 6 are remarkably similar. A small change in analysis technique or time-series length might therefore change the order in which these EOFs appear. Sure enough, if we consider only the last 110 years ( $a$ in the figure, case 2), and inspect EOFs 3 to $6(b)$, we notice that EOF 6 (dashed line) is in quadrature with EOF 3 (dotted line), forming a 20-30-year oscillatory pair, whereas with the full 130 -year time-series, it is EOFs 3 and 4 which are in quadrature (Fig. $1 b$ of ref. 1). Thus, removing the first 20 years of the time-series causes EOFs 4 and 6 to exchange positions in the eigenvalue rank-ordering, through a very slight change in their respective eigenvalues.

Ghil and Vautard impose a Toeplitz structure on the lag-covariance matrix. We are unsure if this is advisable, as it imposes symmetries on the problem which may not be present in a short data series. But if we do the same ( $a$, case 3 ), we can make EOF 4 and EOF 6 swop
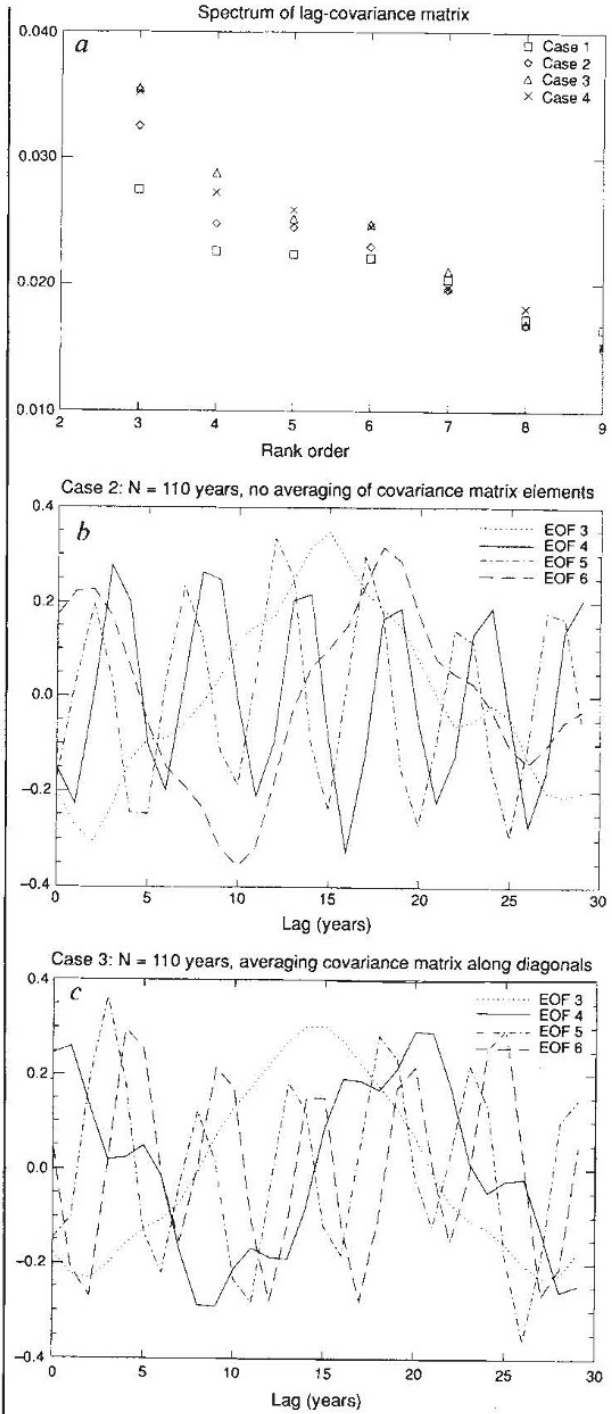

back again $(c)$. If we consider only the last 90 years of the series, they reexchange. If the rank order of these EOFs is so sensitive to details of the analysis, the order itself cannot have any physical significance. In dealing with such a short time-series, the possibility that results may be a consequence of a few anomalous decades, or an artefact of time-series and window lengths, must always be considered carefully. But Elsner and Tsonis have definitely failed to prove their case against the 20-year oscillation.

Department of Physics,

MYLES R. ALLEN

PETER L. READ

University of Oxford,

Parks Rd,

Oxford OX1 3PU, UK

Department of Engineering,

University of Warwick,

Coventry CV4 7AL, UK

1. Ghil, M. \& Vautard, R. Nature 350, 324-327 (1991).

2. Elsner, J. B. \& Tsonis, A. A. Nature 353, 551-553 (1991).

3. Houghton, et at. Climate Change: the IPCC Scientific Assessment (Cambridge University Press, 1990).

NATURE · VOL $355 \cdot 20$ FEBRUARY 1992 